\title{
La gamificación en juego: percepción de los estudiantes sobre un escape room educativo en el aula de español como lengua extranjera
}

\author{
Cristina Martín-Queralt ${ }^{1}$ iD, Jaume Batlle-Rodríguez ${ }^{2}$ \\ Artículo de innovación. Recibido: 25/08/2020. Aceptado: 23/10/2020. Publicación avanzada: 05/12/2020. Publicado: 04/01/2021.
}

Resumen

INTRODUCCIÓN. Los escape rooms son actualmente un entretenimiento muy popular y se ha demostrado, además, que ofrecen numerosos beneficios en otros campos como la enseñanza de lenguas extranjeras. Dentro del ámbito de la gamificación, los juegos de escape se comprenden como adecuados para paliar actitudes negativas y promover la comunicación, el trabajo en equipo, la cooperación y el desarrollo de un aprendizaje significativo acorde a las necesidades e intereses del alumnado.

MÉTODO y OBJETIVOS. Este estudio, de carácter exploratorio, tiene como objetivos conocer qué valoración hacen los alumnos sobre un escape room educativo tras participar en él y qué elementos les han suscitado más aprendizaje en el marco de la enseñanza de español como lengua extranjera (ELE), y además pretende poner de manifiesto el valor de los escape rooms como estrategia metodológica adecuada para la enseñanza-aprendizaje de ELE.

RESULTADOS. El análisis de los cuestionarios de percepción demuestra que los alumnos valoran positivamente el escape room educativo como herramienta y como propuesta didáctica para la enseñanza-aprendizaje de ELE.

CONCLUSIÓN. Los alumnos valoran positivamente la participación en la experiencia didáctica y consideran que han aprendido significativamente, sobre todo en relación con el desarrollo de la comprensión y los contenidos tratados sobre la cultura hispanoamericana.

Palabras clave

Español como lengua extranjera; Gamificación; Escape room educativo; Percepción del alumnado.

Datos de los autores

${ }^{1}$ Universitat de Barcelona, España. Profesora de inglés y español como lenguas extranjeras. Sus principales líneas de investigación se centran en la gamificación, las TIC y la creación de propuestas didácticas y de materiales. Contacto para la correspondencia: cmartinqueralt@gmail.com

${ }^{2}$ Universitat de Barcelona, España. Es doctor en Didáctica de la Lengua y la Literatura por la Universidad de Barcelona. Actualmente es profesor asociado en el departamento de Educación Lingüística y Literaria de la Facultad de Educación de dicha universidad. También es profesor colaborador en el Máster en Formación de Profesores de Español como Lengua Extranjera (UNIBA) y en el Posgrado en Formación de Profesores de Español como Lengua Extranjera (IL3-UB).

\section{Referencia recomendada}

Martín-Queralt, C., y Batlle-Rodríguez, J. (2021). La gamificación en juego: percepción de los estudiantes sobre un escape room educativo en el aula de español como lengua extranjera. REIRE Revista d'Innovació i Recerca en Educació, 14(1), 1-19.

https://doi.org/10.1344/reire2021.14.132195

(C) 2021 Los autores. Este artículo es de acceso abierto sujeto a la licencia Reconocimiento 4.0 Internacional de Creative Commons, la cual permite utilizar, distribuir y reproducir por cualquier medio sin restricciones siempre que se cite adecuadamente la obra original. Para ver una copia de esta licencia, visite https://creativecommons.org/licenses/by/4.0/ 
La gamificación en juego: percepción de los estudiantes sobre un escape room educativo en el aula de español como lengua

\section{Títol (català)}

La ludificació en joc: la percepció dels estudiants sobre un escape room educatiu a l'aula d'espanyol com a llengua estrangera

\section{Resum}

INTRODUCCIÓ. Els escape rooms són actualment un entreteniment molt popular i s'ha demostrat, a més a més, que ofereixen nombrosos beneficis en altres camps com l'ensenyament de llengües estrangeres. Dins l'àmbit de la ludificació, els jocs d'escapada es consideren adequats per pal-liar actituds negatives i promoure la comunicació, el treball en equip, la cooperació i el desenvolupament d'un aprenentatge significatiu d'acord amb les necessitats i els interessos de l'alumnat.

MÈTODE i OBJECTIUS. Aquest estudi, de caràcter exploratori, té com a objectius conèixer quina valoració fan els alumnes després de participar en un escape room educatiu i quins elements els han suscitat més aprenentatge dins del marc de l'ensenyament d'espanyol com a llengua estrangera (ELE), i pretén posar de manifest el valor dels escape rooms com a estratègia metodològica adequada per a l'ensenyament i l'aprenentatge d'ELE.

RESULTATS. L'anàlisi dels qüestionaris de percepció demostra que els alumnes valoren positivament l'escape room educatiu com a instrument i com a proposta didàctica per a l'ensenyament-aprenentatge d'ELE.

CONCLUSIÓ. L'alumnat valora positivament la participació en l'experiència didàctica i considera que ha après significativament, sobretot en relació amb el desenvolupament de la comprensió i dels continguts treballats de cultura hispanoamericana.

Paraules clau

Espanyol com a llengua estrangera; Gamificació; Ludificació; Escape room educatiu; Percepció de l’alumnat.

\section{Title (English)}

Gamification: students' perceptions of an educational escape room in the context of learning Spanish as a foreign language

\section{Abstract}

INTRODUCTION. Recently, escape rooms have become a popular source of entertainment and they also have been shown to offer many benefits in many areas of education, including foreign language teaching. Inside gamification, escape games can alleviate negative attitudes and promote communication, teamwork, cooperation, and the development of a meaningful learning process in accordance with the students' needs and interests.

METHOD and OBJECTIVES. This exploratory paper assesses students' impressions of an educational escape room experiment in which they had taken part, and aims to identify the elements that particularly fostered learning in the Spanish as a Foreign Language (SFL) context. The paper stresses the value of escape rooms as a useful methodological strategy in the SFL context.

RESULTS. The analysis of the questionnaires shows that students had a positive impression of the educational escape room as a teaching and learning tool in SFL.

CONCLUSION. Students expressed positive evaluations of their participation in this teaching experience. They felt that they had made significant progress, especially with regard to the development of their comprehension skills and also their understanding of Spanish culture.

\section{Keywords}

Spanish as a foreign language; Gamification, Educational escape room; Students perceptions. 


\section{Introducción}

La voluntad de establecer acciones didácticas en la búsqueda de una mejora de la enseñanza-aprendizaje ha llevado a muchos profesores a buscar estrategias metodológicas innovadoras que superen las maneras estandarizadas de trabajar en el aula. Una de esas estrategias es la conocida como gamificación (Kapp, 2012; Pujolà y Herrera, 2018; Werbach y Hunter, 2012), la cual se fundamenta en el uso de elementos y estrategias propias del juego en contextos que no son propiamente lúdicos, como el de la enseñanza. En el ámbito de la educación, la gamificación ha tenido un fuerte impacto (Ortiz-Colón et al., 2018), dando pie a numerosas propuestas didácticas que, con mayor o menos éxito, han intentado implementar la gamificación a diversos contextos educativos (Lee y Hammer, 2011; Piñero, 2019). En el ámbito investigativo, algunos trabajos se han focalizado en analizar cómo los profesores construyen sus propuestas didácticas gamificadas, atendiendo al uso de la narrativa (Batlle et al., 2018), cómo se relacionan las diferentes actividades de aprendizaje de la lengua (Batlle y Suárez, 2019) o cómo se atiende a los elementos propios de la gamificación (Pujolà et al., 2017). En la enseñanza-aprendizaje de lenguas extranjeras en concreto, la investigación sobre la implementación de acciones gamificadas es todavía incipiente, teniendo en cuenta la complejidad que entraña la realidad didáctica gamificada, así como las diferentes realizaciones que se pueden construir a partir de los parámetros propios de la gamificación.

Dentro de estas realizaciones didácticas gamificadas, destacan los escape rooms educativos. Este tipo de gamificación recoge algunas de las características de los escape rooms, actividades de carácter lúdicos caracterizadas por que un grupo de personas deben intentar salir de un espacio determinado en un tiempo concreto, y las aplica al aula, muchas veces considerando ese espacio como un lugar cerrado del que hay que salir, pero planteado también como un reto por conseguir en un determinado lapso de tiempo. Los escape rooms educativos son un tipo de propuesta gamificada innovadora, por lo que pocos han sido los estudios que se han acercado a valorar las implementaciones de este tipo de propuestas didácticas (Fotaris y Mastoras, 2019). Aunque si bien esto es cierto, existen algunas propuestas de escape room en el ámbito de la educación: Dimitrova (2019); Peleg et al. (2019); Eukel y Morrell (2020) e incluso con propuestas que incorporan elementos tecnológicos de última generación: la realidad virtual (Yeasmin y Abdulrahman, 2020). Con la intención de contribuir al conocimiento de los escape rooms educativos, el estudio que presentamos con estas líneas aborda la percepción de los estudiantes que participaron en una experiencia práctica fundamentada en este tipo de gamificación. En concreto, este estudio pretende determinar qué valoración hacen los alumnos tras participar en un escape room educativo y qué elementos les han suscitado más aprendizaje, teniendo en cuenta que esta es una experiencia práctica en el ámbito de la enseñanza-aprendizaje de español como lengua extranjera. Con ello, se pretende arrojar luz sobre el valor de los escape rooms educativos como estrategia metodológica adecuada para la enseñanza-aprendizaje de español como lengua extranjera.

\section{Marco teórico}

Aprender y jugar van de la mano y resulta interesante destacar que el uso de dicho elemento lúdico en la educación no es un descubrimiento reciente (Becker, 2017). De hecho, es una estrategia habitual para trabajar diferentes cuestiones lingüísticas, por ejemplo, como el juego del ahorcado para aprender vocabulario, entre muchos otros. Por ello, el juego ha sido y sigue siendo un elemento clave para la educación y el aprendizaje (Donald, 2001; Murray, 2006; Piaget, 1952). Sin embargo, el término juego (Becker, 2017; Huizinga, 1972) incluye una gran variedad de opciones que no sirven para un solo uso u objetivo, aunque mantenga una serie de especificaciones comunes. Asimismo, la realidad educativa ha 
utilizado elementos y especificaciones propias del juego que van más allá de tal actividad. Dos ejemplos de ello son el juego serio y la gamificación (Becker, 2017; Pujolà, 2019; Rodríguez y Santiago, 2015, entre otros).

A partir de la utilidad del juego dentro del ámbito educativo nace el concepto de juego serio (Abt, 1970). Mientras que el juego tiene como objetivo principal el entretenimiento, el juego serio se focaliza en el aprendizaje, a pesar de mantener los elementos lúdicos y de ocio. Según Kapp et al. (2014), los juegos serios o juegos educativos se basan en la metodología de "aprender jugando" y, tal y como apunta Pujolà (2019), suelen ser muy habituales en diferentes áreas como las matemáticas, la física o la enseñanza de lenguas.

A diferencia del anterior, la gamificación (o ludificación) (Deterding et al., 2011; Marín y Hierro, 2013; Ramírez, 2014, entre otros) es una estrategia metodológica fundamentada en el uso de elementos propios del juego en contextos no propiamente lúdicos. Es un concepto que relaciona el juego y el ámbito educativo, pero incluye una serie de elementos (Werbach y Hunter, 2012) que se distingue del juego serio por el hecho de no haber sido concebido como juego previamente y por comprenderse más como una capa a partir de la cual se realiza una serie de acciones (Pujolà, 2019). Asimismo, la gamificación pretende crear propuestas que sean adecuadas para grupos de aprendices con intereses, necesidades y habilidades muy variadas. Cada individuo es diferente y único, por lo que podrá demostrar dichas habilidades, capacidades o conocimientos adquiridos de formas muy diversas. Por este motivo, Nicholson (2012) propone el término gamificación significativa. Nicholson (2012) afirma que a la hora de diseñar propuestas de gamificación es necesario que estas propuestas permitan a los estudiantes tener la libertad de elegir de qué manera van a mostrar su mejora y sus conocimientos para que sean ellos mismos los que fijen sus propios objetivos y logros y, así, conseguir que enseñen su progreso de forma más significativa para ellos. Por su parte, McGonigal (2011) y García et al. (2018) defienden la idea de que el proceso de aprendizaje no se limita exclusivamente al contenido curricular, sino que este también está relacionado inevitablemente con el crecimiento personal, ya sea de carácter social o emocional.

Bajo los parámetros que acabamos de describir se sitúa lo que se conoce como escape room educativo. Esta práctica docente se define como un juego de acción real por equipos en el que los jugadores deben descubrir pistas, resolver enigmas y superar pruebas en una o más salas para lograr un objetivo específico - suele ser salir de la habitación - en un tiempo limitado (Nicholson, 2015). Además, otra característica importante es la del trabajo en grupo, la cooperación y la colaboración entre los participantes para conseguir el objetivo final.

El origen de este concepto tuvo lugar en Japón donde el año 2007 Takao Kato creó el Real Escape Game (REG) basado en el juego "point and click" en el que el jugador tenía que escapar de una habitación tras abrir una serie de candados y resolver unos enigmas y puzzles. Después de convertirse en un fenómeno en Asia, esta idea llegó a manos de los estadounidenses, que fundaron la primera empresa de escape room en América "Puzzle Break" en 2012. Después, los húngaros fueron los siguientes en unirse y crearon el primer juego de escapismo de Europa: Parapark (Corkill, 2009; Nicholson, 2015). Dentro del ámbito educativo, los escape rooms educativos se basan en la idea de los escape rooms lúdicos, pero se enmarcan en un contexto de aprendizaje y tienen unos objetivos académicos específicos (Veldkamp et al., 2020). Los escape rooms educativos se pueden diseñar para cualquier perfil de estudiante y nivel de estudios, siempre y cuando se adapte a las necesidades del grupo (Sánchez, 2018).

La relación entre escape room educativo, juego serio y gamificación es compleja. Según Kapp (2012) ambos conceptos, juego serio y gamificación, comparten ciertos objetivos, tales como la solución de problemas, 
la búsqueda de motivación de los estudiantes y la promoción del aprendizaje a partir de técnicas propias del juego. Kapp (2012) considera que los juegos serios son un tipo de gamificación, puesto que los objetivos se centran en el aprendizaje de cuestiones más concretas y a un nivel más profundo. Así pues, observamos que hay una relación muy estrecha entre el concepto de escape room educativo, la gamificación y los juegos serios, puesto que los juegos de escape pretenden motivar a los alumnos, captar su interés, adaptarse a sus necesidades, desarrollar la autonomía de los aprendices y, así, favorecer un proceso de aprendizaje más significativo. Dichos objetivos coinciden con los que estipula la gamificación, por lo que a través de los elementos de la gamificación se podrán lograr todos los puntos mencionados anteriormente. Además, los escape rooms sirven para cumplir con los objetivos académicos establecidos, por lo que la meta no es la diversión per se, sino el trabajo del contenido curricular a través del juego serio, siguiendo la filosofía de "aprender jugando" (Aldrich, 2005; Pujolà, 2019).

Si bien es cierto que el concepto de escape room es bastante conocido, pocos han sido los estudios que se han acercado a tal realidad lúdica (Fotaris y Mastoras, 2019; Majuri et al., 2018). Por ejemplo, Clarke et al. (2017) afirman que los escape room hacen aumentar la motivación de los estudiantes, así como su pensamiento creativo (Williams, 2018) y su capacidad de liderazgo (Wu et al., 2018). Diago Nebot y Ventura Campos (2017), por su parte, defienden que los juegos de escape motivan a los alumnos, además de provocar que estos hagan uso de sus habilidades al máximo nivel. Stone (2016) identifica que los alumnos desarrollan también las habilidades sociales, la cooperación y el trabajo colaborativo, mientras que Nicholson (2015) afirma que los escape rooms provocan que los alumnos desarrollen una gran variedad de habilidades y un aprendizaje más significativo.

Sin embargo, hasta la fecha, pocos estudios se han desarrollado con la intención de conocer la percepción de los estudiantes con respecto a la participación en un escape room educativo. Santos et al. (2019), tras la implementación de un escape room en el ámbito de la Didáctica de la Química, observan que los alumnos valoran como aspectos positivos de la práctica gamificada el hecho de que se aprenda jugando, el desarrollo de la creatividad, el trabajo en equipo y que se fomente un aprendizaje centrado en el alumno. Por el contrario, los estudiantes valoran negativamente la experiencia del escape room debido a la dificultad de las actividades, la generación de frustración derivada de ello y el trabajo bajo la presión derivado de la temporización de la actividad. Por su parte, Hermanns et al. (2018), desarrollan un escape room para la asignatura de farmacología de los estudios de enfermería. Los alumnos participantes, mayoritariamente, consideraron la propuesta didáctica como valiosa y divertida, y que les permitió llevar a cabo un aprendizaje adecuado. Asimismo, los retos propuestos en la secuencia didáctica se valoraron positivamente, así como el trabajo en equipo. Sin embargo, el hecho de no lograr llegar al objetivo final a tiempo y, por lo tanto, no completar la actividad, les causó frustración y ansiedad. En la misma línea, López-Pernas et al. (2019), tras la implementación de un escape room educativo en un curso de programación, indican que los alumnos percibieron la experiencia educativa como muy positiva, como una experiencia muy divertida y poco estresante. Asimismo, valoraron la experiencia didáctica como más adecuada para desarrollar su aprendizaje que una clase convencional. Moura y Lourido Santos (2019), en un escape room educativo en el ámbito de las matemáticas y la literatura, indican que los alumnos estuvieron encantados por participar en tal experiencia educativa, no encontraron las actividades muy complejas y les pareció muy adecuada para fomentar el trabajo en equipo y la interacción entre ellos, aunque alguno de ellos valoró negativamente la presión impuesta por el tiempo. También en el ámbito de la literatura, Martínez-Borobio y Nakova-Katileva (2020) realizan un estudio centrado en las valoraciones de un escape room educativo. En su caso, los estudiantes también valoran muy positivamente la experiencia didáctica e inciden en que el trabajo en equipo y las tareas les han sido beneficiosas y significativas. Además, el estudio también se centra en las valoraciones de los alumnos en relación con su desarrollo competencial. Estos valoran muy 
positivamente la experiencia, sobre todo en relación con el desarrollo de la comprensión lectora, habilidad comunicativa más presente en el escape room propuesto.

Los estudios a los que se ha aludido hacen referencia a la valoración de los estudiantes acerca de los escape rooms educativos. Siguiendo esta línea de investigación, este estudio focaliza su atención no solo en la valoración que los estudiantes hacen del escape room educativo como experiencia didáctica, sino también en cómo valoran el tratamiento de los diferentes aspectos involucrados en el aprendizaje de español como lengua extranjera. En concreto, este trabajo se propone analizar la valoración que una serie de alumnos de español como lengua extranjera en un contexto de inmersión hacen de un escape room educativo tras participar en él. Derivadas de los objetivos que acabamos de especificar, nos planteamos las siguientes preguntas de investigación: ¿Qué valoración de los escape rooms educativos hacen una serie de alumnos de español como lengua extranjera? ¿Qué percepción tienen del aprendizaje de español como lengua extranjera que se ha podido derivar de la participación en un escape room educativo?

\section{Método}

Esta investigación, de carácter exploratorio, se deriva de la creación y pilotaje de una propuesta didáctica bajo los parámetros de los escape rooms educativos intitulada Diamante Hispano.

\subsection{Materiales}

La propuesta didáctica en cuestión de enfoque comunicativo consta de ocho actividades y tiene una duración estimada de 1 hora y 20 minutos. La narrativa de la propuesta es la siguiente: 5 países hispanohablantes - México, Chile, Colombia, Cuba y España - son los posibles paraderos de uno de los mayores tesoros de la historia de Hispanoamérica: el diamante hispano, una joya de un valor incalculable que ha pertenecido a algunas de las personas más importantes de la historia de dichos países. Un ladrón ha robado el patrimonio, pero no se sabe dónde lo ha escondido. Las policías de todos los países hispanohablantes necesitan ayuda para encontrar tanto al ladrón como al tesoro. La escuela propone a los alumnos como equipo de investigación para resolver el caso y dará una recompensa si lo resuelven con éxito. Sin embargo, el equipo de investigación no tiene tiempo que perder: si no encuentran al ladrón y al diamante en 1 hora y 20 minutos, el ladrón ya habrá huido con la joya y jamás se podrá volver a recuperar. Como se observa de la descripción de la narrativa del juego, el objetivo que tienen los alumnos es lograr encontrar al ladrón y el diamante en un espacio de tiempo determinado. A diferencia de los escape rooms lúdicos, que implican tener que salir de un espacio, aquí la lucha contra el reloj no es por salir, sino por lograr el objetivo del juego.

En la secuencia de ocho actividades se trabajan todas las destrezas -comprensión escrita, comprensión auditiva, expresión escrita e interacción oral-, así como también cuestiones gramaticales, culturales y de vocabulario. Además del contenido meramente lingüístico, se pretende potenciar e incluir el uso de las nuevas tecnologías, empleando recursos como el uso de material audiovisual creado específicamente para las actividades, el uso de códigos QR, así como también aplicaciones que utilizamos en nuestro día a día como, por ejemplo, Google Maps, el correo electrónico y Youtube. Cabe destacar que, todas las actividades contribuyen a resolver el misterio del diamante hispano y, por lo tanto, a conseguir el objetivo final del room escape, tomando como base el uso de elementos del juego propios de la gamificación. El uso de dichos elementos resulta innovador, puesto que se aleja de un modelo educativo más tradicional y su presencia en la 
propuesta Diamante Hispano es clave para fomentar la motivación, la participación y el trabajo colaborativo por equipos, además de procurar un proceso de aprendizaje significativo de los estudiantes.

\subsection{Participantes}

El escape room educativo se llevó a la práctica en una escuela de español como lengua extranjera en Barcelona (España) durante el otoño de 2019. La propuesta se llevó al aula por partida doble. En un primer pilotaje, participaron 11 estudiantes de diversos países: Francia, Alemania, Italia, Hungría, Estados Unidos, Canadá, Reino Unido y Polonia. De los 11 participantes, 8 eran mujeres y 3 eran hombres, todos de entre 18 y 24 años. La edad media del grupo era de 21 años.

En el segundo pilotaje el grupo se componía de 12 estudiantes de diferentes países Francia, Suiza, Bélgica, Holanda, Noruega, Estados Unidos, Taiwán e Italia. De los 12 participantes 7 eran mujeres y 5 hombres, todos de entre 19 y 25 años. La edad media del grupo era de 22 años.

Según el programa de curso de la escuela, ambos grupos tenían un nivel A2 de español (Consejo de Europa, 2002). Ambos pilotajes fueron impartidos por una de las autoras de este trabajo. Cabe destacar, a este respecto, que la autora de este trabajo que impartió las sesiones no era la docente habitual.

\subsection{Método}

Justo después de la sesión de clase en la que se implementó el escape room educativo, se pasó a los alumnos un cuestionario. El cuestionario fue creado específicamente para la ocasión por la autora de la propuesta y constaba de 14 preguntas -4 en formato escala de Likert, 7 de respuesta abierta y 3 de elección múltiple- que permitían evaluar diferentes aspectos: primeramente, conocer la experiencia previa de los participantes en el marco de los escape rooms, así como también la valoración de la experiencia vivida por parte de los participantes en relación con los diferentes elementos clave que conforman el juego de escape - los aspectos lingüísticos y el uso de la lengua, el uso de los elementos del juego y de la gamificación y, por último, el uso de las TIC—; finalmente, se incluyó una pregunta de valoración global, para obtener un compendio general de sus opiniones sobre la sesión.

Con el fin de obtener unos resultados lo más completos posibles, en el cuestionario se incluyeron preguntas tanto de carácter cuantitativo, cuyos resultados se representan a través de gráficos, como también preguntas cualitativas, cuyo análisis se basa en la interpretación de las respuestas registradas. La congruencia entre ambos tipos de datos nos permite determinar la validez de estos $y$, por consiguiente, alcanzar unas conclusiones sólidas y fundamentadas en relación con las creencias de los estudiantes que han sido partícipes de esta experiencia. Los resultados expuestos en esta investigación se derivan de las preguntas que se formularon para conocer la valoración general de los estudiantes sobre la propuesta didáctica en la que participaron, así como su percepción acerca del aprendizaje de español como lengua extranjera implicado en ella. 


\section{Resultados}

Dado que la investigación de carácter exploratorio que exponemos en estas líneas tiene el doble objetivo de presentar la valoración general de una serie de alumnos de español como lengua extranjera con respecto a una propuesta de escape room educativo, así como del aprendizaje derivado de su participación en la misma, se ha optado por presentar los resultados del análisis en dos subapartados diferenciados. El primero analizará la valoración del escape room por parte de los estudiantes, mientras que el segundo se centrará en presentar el análisis de las respuestas del cuestionario de valoración centradas en el aprendizaje de español como lengua extranjera implicado en la propuesta.

\subsection{Valoración del escape room educativo por parte de los estudiantes}

Como se ha comentado previamente, la propuesta didáctica Diamante Hispano tenía la intención de llevar al aula de español como lengua extranjera un escape room educativo. Ante esta nueva estrategia metodológica, queríamos conocer de primera mano cuál fue la percepción de la propuesta. Para ello, se propusieron tres preguntas en el cuestionario de valoración, todas ellas centradas en determinar la experiencia global de los participantes. Cabe mencionar que este estudio estadístico, basado en datos cuantitativos, tiene la intención de identificar tendencias, sin pretender ofrecer resultados concluyentes debido a las limitaciones del tamaño de la muestra analizada.

En este sentido, debemos destacar que, como se observa en la Figura 1, una gran mayoría de estudiantes $(n=19)$ cualificó como muy positiva $(n=10)$ o positiva $(n=9)$ la experiencia, mientras que una minoría lo valoró como poco positiva $(n=3)$ o con un término medio $(n=1)$ y ningún estudiante la valoró como negativa. Así pues, como se desprende de las respuestas de los alumnos, en líneas generales la valoración de los estudiantes de la propuesta didáctica fue muy positiva.

\section{Figura 1}

Pregunta 14: Tu opinión global de la experiencia ha sido...

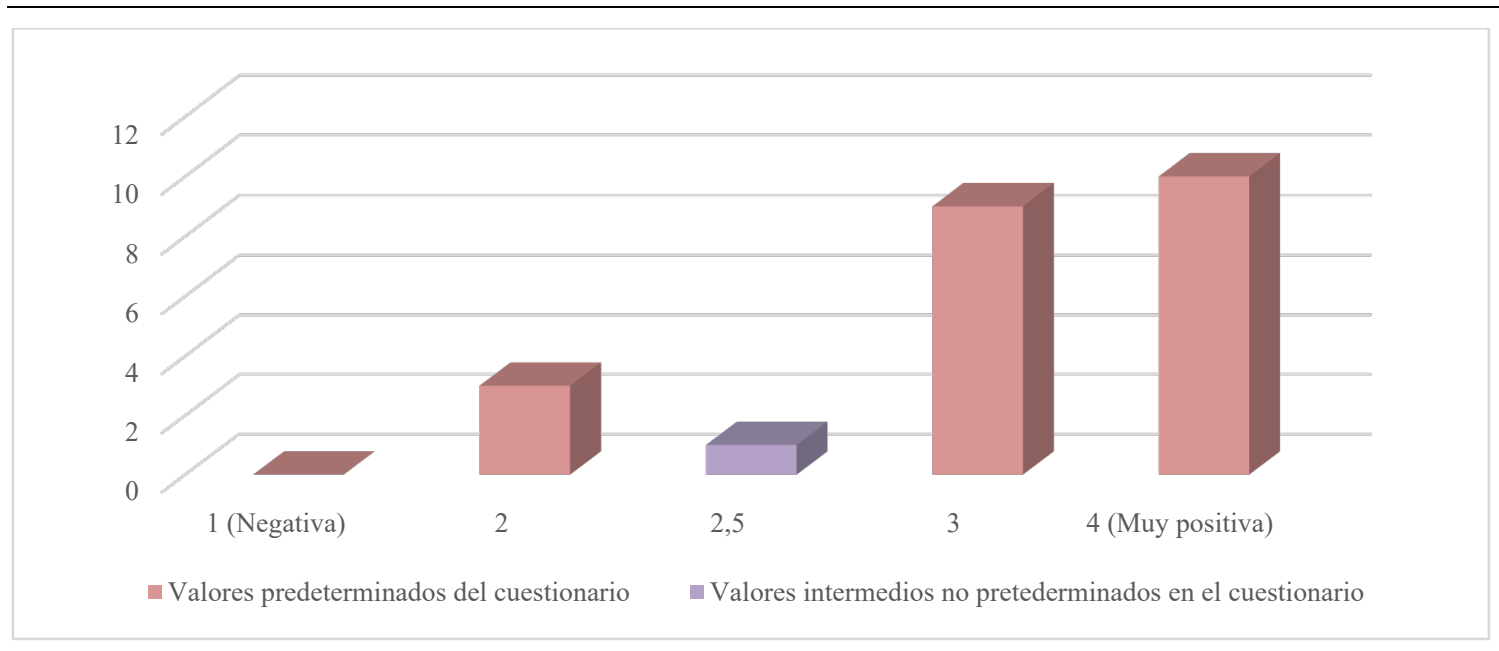

Por otro lado, para recoger la percepción de los estudiantes sobre la propuesta didáctica, se les formuló a los alumnos dos preguntas más: una primera centrada en si consideraban que la clase en la que habían participado había sido divertida y otra con la intención de que explicaran si les había gustado el escape room. Las dos preguntas mantienen ciertas similitudes, aunque estaban pensadas 
para que la primera fuera una valoración general -juego y clase de español como lengua extranjera-, mientras que la segunda focalizaba la atención exclusivamente en el hecho lúdico, en el escape room.

De las 23 respuestas ofrecidas por parte de los alumnos para valorar si la propuesta didáctica había sido divertida, 18 estudiantes respondieron afirmativamente, mientras que únicamente cinco estudiantes respondieron negativamente, resultados que concuerdan con lo expresado en la pregunta sobre la valoración global. En concreto, las razones por las cuales respondieron de manera afirmativa tienen que ver con la propia dinámica de la actividad. Por un lado, muchos estudiantes consideraron divertida la propuesta porque era diferente a lo que solían hacer en sus clases de español, mientras que algunos otros la valoraron positivamente debido al tratamiento de los contenidos propuestos o a la dinámica de grupos establecida. Por ejemplo, algunos valoraron la experiencia como una actividad muy diferente a sus rutinas habituales en las clases de español. En concreto, un estudiante comentaba que "ha sido divertida y también instructiva" (estudiante 10, pilotaje 1).

Otros, en la misma línea, valoraban el hecho de que el escape room educativo fuera una manera de salir de la rutina, puesto que "fue muy diferente de cada actividad ha hecho en las clases de español" (estudiante 2, pilotaje 1). En este sentido, el escape room implica un aprendizaje diferente: "me gusta aprender en otra forma y manera" (estudiante 4, pilotaje 1). La percepción de divertido también ha sido asociada por algunos estudiantes al hecho de haber jugado. Así, un estudiante comenta que la propuesta didáctica le ha parecido divertida porque "es muy diferente a lo normal y fue como un juego" (estudiante 5, pilotaje 2). Además de valorar positivamente la propuesta didáctica por ser diferente y salir de la rutina de las clases habituales, algunos otros estudiantes la valoraron positivamente por el contenido tratado y por la forma de tratarlo. Así, se valora positivamente la propuesta didáctica "porque habla del mundo y de países de Sudamérica" (estudiante 5, pilotaje 1) o "es una buena forma de practicar el español" (estudiante 1, pilotaje 1). Por otro lado, otros estudiantes también valoran positivamente la propuesta didáctica en relación con aspectos propiamente metodológicos como el planteamiento propuesto para resolver ejercicios, todos ellos en grupos (estudiantes 6 y 7, pilotaje 1; estudiante 2, pilotaje 2) y a partir de una dinámica que implicaba la necesaria participación de todos los estudiantes. Ello implicaba que la propuesta didáctica creara un ambiente agradable (estudiante 1, pilotaje 2).

Sin embargo, como apuntábamos, no todos los estudiantes valoraron como divertida la propuesta. Las razones argüidas para ello radicaban en el excesivo uso del teléfono móvil (estudiantes 8 y 9 , pilotaje 1) o al hecho de que el ritmo de las actividades no fuera el óptimo "porque tenías que esperar a los otros" (estudiante 11, pilotaje 2). En el primer caso, los alumnos inciden en el excesivo uso del teléfono debido a que muchas de las actividades que se proponían en la propuesta didáctica se fundamentaban en un aprendizaje móvil, como el uso de lectores de código QR o el visionado de vídeos en Youtube. En el segundo caso, es interesante destacar que el hecho negativo está en una realidad propia de los escape room: el grupo se considera un todo y, como tal, todos logran el objetivo del juego - salir del espacio en el que están - o no. En la propuesta didáctica planteada, los estudiantes, en grupos, tenían que realizar diferentes acciones, pero todas estas acciones se interrelacionaban y se tenían que realizar al mismo tiempo para ir avanzando en el juego. Este hecho propició que, en alguna ocasión, algún grupo de alumnos tuviera que esperar a otros grupos para continuar avanzando en el juego, detalle que desmotivó concretamente a esta estudiante. Estas son 
apreciaciones puntuales que permiten observar algunas discrepancias; no obstante, el sentir general de los dos grupos de alumnos con los que se pilotó la propuesta fue mayoritariamente positivo.

En relación con la pregunta sobre la valoración general de la propuesta como actividad didáctica gamificada, las valoraciones positivas también fueron las más habituales. Los mismos estudiantes que valoraron la propuesta como divertida en la anterior pregunta consideraron que la experiencia del escape room educativo fue positiva, sobre todo porque supuso una experiencia divertida. Sin embargo, las razones por las cuales los alumnos consideraron positivo el escape room no solo se fundamentan en cuestiones propias de las dinámicas de clase, como trabajar en grupos (estudiante 2, pilotaje 1) o en el contenido propio del aprendizaje de español como lengua extranjera, sino que los alumnos también ponen el foco de atención en aspectos propios del juego. Así, se valora positivamente el hecho de que fuera "emocionante" (estudiante 6, pilotaje 1) o que la propuesta se fundamentara en "resolver un misterio" (estudiantes 4 y 7 , pilotaje 1). Por su parte, los estudiantes que valoraron negativamente la propuesta, como en la pregunta anterior, adujeron que había demasiadas actividades en las que se dependía del móvil para su realización o que la dinámica implicaba que se tuviera que esperar a los grupos más lentos para avanzar en la propuesta gamificada (estudiante 6, pilotaje 2).

\subsection{Percepción del aprendizaje por parte de los estudiantes}

El otro foco de atención de este estudio está en la valoración que los estudiantes hicieron del escape room educativo como propuesta didáctica para el aprendizaje de español como lengua extranjera. En el cuestionario final, cuatro preguntas fueron formuladas con la intención de extraer información en esta línea. Concretamente, estas preguntas estuvieron centradas en las razones por las cuales los alumnos pensaban que la propuesta didáctica les había ayudado o no a mejorar su español, cuánto pensaban que habían mejorado, qué actividad les había gustado más y, por último, qué actividad pensaban que les había ayudado más a mejorar en su español, esto último razonándolo adecuadamente. Cabe reiterar que la información proporcionada en este apartado se analiza desde un punto de vista exploratorio.

Con respecto a la valoración general de la propuesta en relación con su mejora del español, las valoraciones de los alumnos presentan 16 respuestas positivas y 7 respuestas negativas. Así, en general, los participantes consideran que han notado mejoría por diversos motivos, aunque los más recurrentes son los centrados en el uso de la lengua: los que se practica la expresión e interacción oral en la lengua objeto de aprendizaje, los audios y los textos, puesto que eran en español, y la práctica de las diferentes formas verbales. En esta línea, hay alumnos que expresan que han hablado mucho (estudiante 8 , pilotaje 2) y que, por ello, "mi expresión oral ha mejorado" (estudiante 7 , pilotaje 1) y que se han sentido muy cómodos "con hablar solamente español con los otros" (estudiante 2, pilotaje 1). Otros alumnos inciden en que han mejorado en aspectos del sistema de la lengua, como en el dominio de la gramática, pero, sobre todo, inciden en que han aprendido muchas palabras nuevas de vocabulario (estudiantes 6 y 11, pilotaje 1; estudiante 11, pilotaje 2). Dichas muestras son evidencias de que muchos de los participantes son conscientes de haber mejorado su español gracias a las actividades propuestas y reconocen que la práctica y los contenidos trabajados han contribuido a un mejor dominio de la lengua objeto de aprendizaje. Por último, cabe destacar que dos estudiantes valoran positivamente el hecho de trabajar con contenidos propios de las variantes hispanoamericanas. Uno de los estudiantes indica que la propuesta le "ha ayudado a mejorar mi conocimiento sobre México", mientras que un segundo afirma que la propuesta didáctica 
le ha permitido mejorar en español "porque te hace mirar y escuchar que (...) en Sudamérica es diferente la comunicación hispanoamericana” (estudiante 5, pilotaje 1).

Por otro lado, encontramos opiniones más bien negativas en relación con la pregunta en cuestión: varios de los participantes coinciden en que no han mejorado su español, mientras que algunos creen que en algunas destrezas pueden haber mejorado, pero que a nivel general no ha sido así. Un participante reitera dicha opinión negativa y argumenta que los ejercicios para practicar la gramática no eran difíciles, por lo que no siente que haya mejorado (estudiante 4, pilotaje 2). Otro participante manifiesta que no ha mejorado en cuanto a la expresión escrita (estudiante 8 , pilotaje 1) porque solo había una actividad y, por consiguiente, no considera haber mejorado. En este caso, a partir de sus valoraciones podemos interpretar que este estudiante asocia la mejora con actividades de producción.

De las respuestas proporcionadas, se desprende que los estudiantes son conscientes de la mejora en destrezas como, por ejemplo, la interacción oral o la comprensión auditiva, puesto que les resulta evidente el uso de los audios y los vídeos y la necesidad de hablar entre ellos para avanzar en el juego y resolver las diferentes actividades. Sin embargo, en otras actividades, no es tan evidente la práctica de algunas destrezas o de ciertos aspectos lingüísticos, como la gramática. Destaca también la percepción positiva por parte de los estudiantes debido al aprendizaje de nuevo vocabulario, a pesar de que no hubiera ninguna actividad explícitamente pensada para el tratamiento del vocabulario. Este hecho nos hace comprender que muchos estudiantes valoran el aprendizaje de español como lengua extranjera como algo no fundamentado tanto como en el desarrollo de una serie de habilidades de comunicación, sino algo más centrado en cuestiones propias del sistema de la lengua.

La pregunta centrada en cuánto creían que habían mejorado incidía específicamente en valorar numéricamente la mejora producida en varios ámbitos. En concreto, en los aprendizajes de gramática, vocabulario y cultura hispanoamericana, además de incidir en la mejora en relación con las comprensiones lectora y auditiva, y la expresión escrita. Como se observa en la Figura 2, la mayoría de los participantes $(n=16)$ cree que han mejorado muy poco $(n=6)$ o poco $(n=10)$ en cuestiones de gramática, aunque también hubo estudiantes que manifestaron un mayor grado de mejoría, al indicar que habían mejorado bastante $(n=4)$ o mucho $(n=2)$.

Respecto al vocabulario, un alto porcentaje de participantes determinó que su mejoría fue entre poca $(n=8)$ y bastante $(n=9)$. Además, aunque en menor medida, también hubo estudiantes que optaron por los valores más extremos, al expresar muy poco aprendizaje $(n=2)$ o mucho aprendizaje $(n=4)$ para determinar su percepción de mejora en el cuanto al léxico. En lo que se refiere a la cultura hispanoamericana, podemos determinar que la gran mayoría de los participantes consideran que su mejoría en este campo fue bastante $(n=11)$ o mucha $(n=4)$, mientras que un número menor de estudiantes especificaron que el aprendizaje en este ámbito había sido poco $(n=4)$ o muy poco $(n=2)$. 
Figura 2

Pregunta 5: ¿Cuánto piensas que has mejorado?

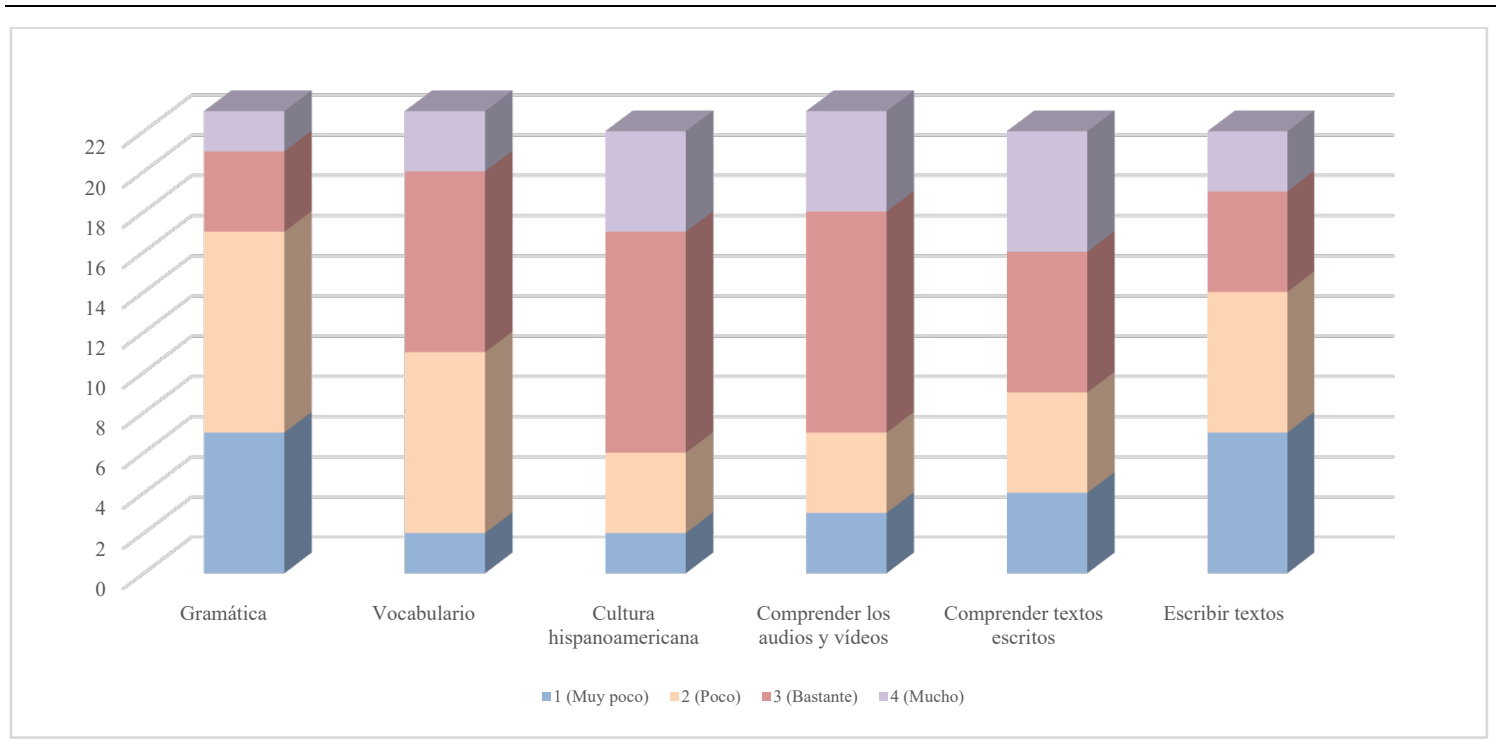

La comprensión auditiva también fue una de las categorías que los participantes puntuaron con valores más altos, dando a conocer que su mejoría había sido notable, puesto que la gran mayoría marcó valores de bastante $(n=11)$ y mucho $(n=5)$ en la escala de Likert. En cambio, los valores de poco $(n=4)$ o muy poco $(n=3)$ fueron marcados por un menor número de estudiantes.

En cuanto a la comprensión escrita, cabe destacar que hubo participantes que se abstuvieron de responder y, por lo tanto, el número máximo es inferior al del resto de categorías. Sin embargo, en el gráfico 2 podemos observar que la opinión de los participantes en relación con la mejoría de dicha destreza está dividida, aunque con una tendencia a que se valore positivamente el trabajo derivado de la propuesta didáctica. Podemos percibir una ligera mayoría en los valores más altos, con una mayor presencia del valor bastante $(n=7)$ en relación con el valor mucho $(n=6)$. La tendencia sigue decreciendo con los valores negativos ( $n=5$ para poco; $n=4$ para muy poco).

Por último, en relación con la expresión escrita podemos determinar que la mayor parte de los participantes considera que su mejoría ha sido poca $(n=7)$ o muy poca $(n=7)$. Sin embargo, también hay muestras de valoraciones más altas ( $n=5$ para bastante; $n=3$ para mucho) por parte de algunos estudiantes. Cabe mencionar que en el gráfico no se recogen datos sobre la expresión y la interacción oral, puesto que no había actividades que tuviesen como objetivo principal el trabajo de dicha destreza, sino que esta se ponía en práctica a lo largo de todas las actividades, así como también en la cooperación y el trabajo en equipo entre los estudiantes y los diferentes grupos.

Las valoraciones de los estudiantes con respecto a cuánto creían que habían mejorado en diferentes aspectos propios de la enseñanza-aprendizaje de español como lengua extranjera muestran una clara tenencia a valorar la propuesta didáctica como adecuada para mejorar en cuestiones de comprensión auditiva y lectora, y aspectos de la cultura americana, aunque también la mejora en el dominio del vocabulario es percibida por parte de los estudiantes. Sin embargo, el trabajo de la gramática o de desarrollo de la expresión escrita es visto por los estudiantes como escaso, por lo que 
no aprecian una mejora sustancial en estos dos aspectos tras la participación en el escape room educativo.

Otro de los aspectos en los que se quiso indagar fue en qué actividades habían sido más provechosas para los alumnos. Sobre ello, se aprecian opiniones muy dispares a la hora de responder a la pregunta en cuestión. El foco de atención de la mayoría de las respuestas está en el elemento lúdico de las actividades. Los alumnos valoran positivamente las actividades, no por el elemento de aprendizaje involucrado, sino por la dinámica lúdica establecida. Por ejemplo, los alumnos destacan las actividades en las que debían obtener un código secreto para abrir una serie de cajas (estudiantes 3 y 5, pilotaje 1), la etapa del final del escape room, relacionada con la investigación en la que debían resolver dónde estaba el ladrón y dónde estaba el diamante (estudiante 11, pilotaje 2) o un crucigrama con el que debían ordenar las letras para saber la ubicación de la siguiente pista (estudiante 2, pilotaje 1). Otros estudiantes destacan que las actividades que más les gustaron fueron aquellas en las que debían "adivinar las ciudades" (estudiante 8, pilotaje 1), relacionar una serie de siluetas de países hispanoamericanos con sus banderas (estudiante 11, pilotaje 1) o relacionar una serie de "imágenes con sus descripciones" (estudiante 4, pilotaje 2). Sin embargo, cuando el foco de atención está en el elemento de aprendizaje involucrado, este suele ser un elemento del sistema de la lengua, como la gramática o el vocabulario (estudiante 2, pilotaje 2). No se suele poner el foco de atención en el hecho de que desarrollaran alguna habilidad comunicativa, ya sea leer, escribir, escuchar o llevar a cabo interacciones orales entre ellos, aunque dos estudiantes destacaron que la actividad que más les había gustado era la de escuchar vídeos "porque está bien para mejorar" (estudiante 5, pilotaje 2).

Las valoraciones en relación con la actividad que consideran que más ha contribuido a su mejora del español también fueron diversas. Hay algunas actividades que predominan frente a otras, principalmente destacan las que tienen como objetivo la práctica de comprensión escrita y oral. Muchos alumnos destacan que les fue muy provechoso "leer los textos y ver los vídeos" (estudiante 6, pilotaje 2) o simplemente "los audios" (estudiantes 3 y 6 , pilotaje 1; estudiantes 1,7 y 9 , pilotaje 2). En este sentido, es interesante destacar las palabras del estudiante 5 (pilotaje 1), quien relaciona las comprensiones auditivas con las variantes hispanoamericanas. Es interesante resaltar que, las opiniones de los participantes para la pregunta en cuestión se centran, sobre todo, en actividades en las que hay una práctica explícita y evidente de la destreza trabajada con material tangible, mientras que, por otro lado, no consideran que hayan mejorado tanto otras destrezas como la interacción oral, a pesar de que hubiese mucha práctica en la gran mayoría de actividades. En este sentido, se percibe que los estudiantes son más conscientes de su mejoría cuando trabajan con materiales tangibles y con destrezas o contenidos que pueden medir a través de la producción física. Es decir, pueden determinar una mayor mejoría al observar que son capaces de producir una frase escrita en los tiempos verbales que se les pide, pero les resulta más imperceptible apreciar dicha mejoría cuando la producen en un canal oral.

\section{Discusión}

En relación con la primera pregunta de investigación, el análisis de las respuestas mediante un cuestionario de percepción administrado a los estudiantes con el objetivo de conocer su opinión sobre el escape room educativo Diamante Hispano nos ha permitido observar que, en líneas generales, la valoración de los alumnos ha sido positiva. La experiencia gamificada, pues, ha resultado ser adecuada para con lo que los 
alumnos esperan en relación con el aprendizaje de español como lengua extranjera. En este sentido, los resultados de este estudio siguen la línea de Hermanns et al. (2018), López-Pernas et al. (2019) o Moura y Lourido Santos (2019), quienes también mostraron que sus escape rooms educativos eran valorados positivamente por los estudiantes. En concreto, en Diamante Hispano se consideró divertida por ser una experiencia didáctica diferente y por la misma dinámica de la actividad, la cual estaba fundamentada en la resolución de un misterio. En relación con las valoraciones negativas, es necesario destacar que no se apreció como aspecto negativo la gestión del tiempo, como se muestra en os estudios de Hermanns et al. (2018) o Moura y Lourido Santos (2019), sino que, en nuestro caso, las apreciaciones negativas estuvieron relacionadas con el excesivo uso de tecnología móvil y con el hecho de que todos los alumnos conformaran un solo grupo, ante lo cual los alumnos que acababan antes con sus tareas tenían que esperar a los que todavía no habían finalizado. Ello nos da a entender que, quizás, uno de los aspectos de gestión compleja que puede tener un escape room educativo está en la gestión de la participación. El hecho de que sean muchos participantes implica la necesidad de tomar una decisión metodológica: plantear tareas para todos en pequeños grupos implica que todos los alumnos estén activos en mayor o menor medida. Sin embargo, un grupo puede acabar antes y, en consecuencia, debe esperar a que el resto de los compañeros finalice para poder pasar a la siguiente prueba del escape room. En cambio, si se propone solo una actividad para todo el grupo, muy supuestamente esta solo será realizada por unos pocos alumnos, por lo que los estudiantes menos participativos o motivados actuarán como meras comparsas en el juego.

En relación con la percepción del aprendizaje implicado, se ha podido comprobar cómo, en general, los alumnos consideran que el escape room educativo propuesto incide positivamente en el desarrollo competencial en el español como lengua extranjera. La valoración positiva por parte de los alumnos en cuanto a su aprendizaje se debe, entre otros aspectos, al valor lúdico de la secuencia didáctica y a las características de las actividades propuestas, pensadas para realizar acciones en las que el uso de la lengua estuviera implicado. En esta línea, los resultados del estudio están en línea con Moreno Campos y Suvires García (2018), quienes también observan que los alumnos perciben que, a través de la gamificación, su desarrollo competencial aumenta. Con ello, se observa que el desarrollo de experiencias didácticas a través de unas actividades basadas en el juego hace que los alumnos consideren que su aprendizaje se desarrolla positivamente.

Asimismo, la valoración positiva en concreto del desarrollo de la comprensión lectora y la comprensión auditiva, así como del conocimiento de aspectos culturales hispanoamericanos concuerda con la caracterización de la propuesta didáctica. En la misma línea que Martínez-Borobio y Nakova-Katileva (2020), los estudiantes que participaron en Diamante Hispano consideran que su aprendizaje se ha desarrollado adecuadamente. Sin embargo, nuestros alumnos no consideraron que se había desarrollado en demasía su conocimiento gramatical ni su habilidad de expresión escrita. Esta apreciación nos da a entender que, si los contenidos gramaticales no se trabajan como elemento central en la propuesta didáctica, los alumnos consideran que no han trabajado mucho la gramática; del mismo modo, el hecho de que no escribieran durante la mayoría del tiempo hizo que los alumnos consideraran que no habían practicado en demasía tal habilidad. Los resultados muestran que las universidades de Cataluña utilizan las RRSS de forma muy variada, sin una comunicación y planificación digital unificada para todas. Aun así, el trabajo desveló que algunas universidades obtuvieron buenos resultados en RRSS en determinadas variables y en cambio otras se situaron en la parte final de todas las variables. 


\section{Conclusiones}

Tal como indicábamos en la introducción del presente artículo, el objetivo principal de este se centraba en la percepción de los estudiantes tras participar en una experiencia práctica basada en una propuesta de un escape room educativo y en cuáles son los elementos que han suscitado más aprendizaje en relación con la enseñanza-aprendizaje de español como lengua extranjera.

En este sentido, podemos observar que, en líneas generales, los estudiantes valoraron de forma positiva o muy positiva la experiencia por diferentes razones, entre las cuales se destacan el componente lúdico, la incorporación de actividades diferentes y que se salen de su rutina habitual en clase, así como por las dinámicas y los materiales empleados durante el desarrollo de la sesión. También hemos podido anotar opiniones no tan positivas, aunque en menor medida. Estas últimas valoraciones se justifican principalmente por el tiempo de espera entre los diferentes grupos y por la menor presencia de actividades en las que se trabajan algunas de las destrezas como, por ejemplo, la gramática o la expresión escrita, puesto que los estudiantes asocian la producción con un mayor aprendizaje. A pesar de la diversidad de opiniones recogidas, a nivel global la propuesta ha contado con un alto porcentaje de aceptación y los alumnos de ambos pilotajes han contribuido a corroborar la utilidad y la eficacia de llevar a cabo propuestas gamificadas como Diamante Hispano al aula de lenguas extranjeras.

Como docentes debemos ser conscientes de la variedad de gustos, preferencias, dificultades, tradiciones, etc., de nuestros alumnos y resulta evidente que es imposible satisfacer a todo el mundo. Sin embargo, las valoraciones extraídas de la experiencia en cuestión han sido positivas en líneas generales, por lo que podemos concluir que, aunque como profesores podamos tener miedo o respeto a utilizar propuestas gamificadas en el aula por ser algo desconocido y ajeno a nuestra zona de confort, merece la pena probarlo y comprobar en primera persona si se percibe una mejora en cuanto a la motivación, el interés, la autonomía, las habilidades sociales, personales y, por supuesto, en el rendimiento académico y en su proceso de aprendizaje.

\section{Referencias}

Abt, C. C. (1970). Serious games. Viking Press.

Aldrich, C. (2005). Learning by doing: a comprehensive guide to simulations, computer games, and pedagogy in e-learning and other educational experiences. Pfeiffer.

Batlle, J., González, V., y Pujolà J. T. (2018). La narrativa como elemento cohesionador de tareas gamificadas para la enseñanza de lenguas extranjeras. Rivista dell'Istituto di Storia dell'Europa Mediterranea, 2(II), 5-160. https://doi.org/10.7410/1357

Batlle, J., y Suárez, M. M. (2019). Secuencias didácticas gamificadas por docentes de LE en formación continua: puntos, insignias y tablas de clasificación. E-AESLA, 5, 43-51.

Becker, K. (2017). Choosing and using digital games in the classroom: a practical guide. Springer. https://doi.org/10.1007/978-3-319-12223-6 
Clarke, S. J., Peel, D. J., Arnab, S., Morini, L., Keegan, H., y Wood, O. (2017). EscapED: a framework for creating educational escape rooms and interactive games to for higher/further education. International Journal of Serious Games, 4(3), 73-86. https://doi.org/10.17083/ijsg.v4i3.180

Consejo de Europa. (2002). Marco común europeo de referencia para las lenguas: aprendizaje, enseñanza, evaluación. Anaya y CVC.

Corkill, E. (2009, 20 de diciembre). Real escape game brings its creator's wonderment to life. The Japan times. https://www.japantimes.co.jp/life/2009/12/20/to-be-sorted/real-escape-game-brings-itscreators-wonderment-to-life/\#.Xe02hvZFzCC

Deterding, S., Dixon, D., Khaled, R., y Nacke, L. (2011). From game design elements to gamefulness: defining "gamification". En Proceedings of the 15th international academic MindTrek conference (pp. 9-15). https://doi.org/10.1145/2181037.2181040

Diago Nebot, P. D., y Ventura Campos, N. (2017). Escape Room: gamificación educativa para el aprendizaje de las matemáticas. Suma+, 85, 33-40.

Dimitrova, G. (2019). Science Escape Room. Edu\&Tech, 10, 306-309. https://doi.org/10.26883/2010.192.1815

Donald, M. (2001). A mind so rare: the evolution of human consciousness. W. W. Norton.

Eukel, H., y Morrell, B. (2020). Ensuring Educational Escape-Room Success: The Process of Designing, Piloting, Evaluating, Redesigning, and Re-Evaluating Educational Escape Rooms. Simulation \& Gaming. https://doi.org/10.1177/1046878120953453

Fotaris, P., y Mastoras, T. (2019). Escape Rooms for Learning: A Systematic Review. En L. Elbaek, G. Majgaard, A. Valente y S. Khalid (eds.), Proceedings of the 13th International Conference on Game Based Learning (pp. 235-243). Academic Conferences and Publishing International Limited.

García, R., Bonilla, M., y Diego, J. M. (2018). Gamificación en la escuela 2.0: una alianza educativa entre juego y aprendizaje. En A. Torres y L. M. Romero (eds.), Gamificación en iberoamérica experiencias desde la comunicación y la educación (pp. 71-96). Universidad Politécnica Salesiana.

Hermanns, M., Deal, B., Campbell, A. M., Hillhouse, S., Brian Opella, J., Faigle, C., y Campbell, R. (2018). Using an "Escape Room" toolbox approach to enhance pharmacology education. Journal of Nursing Education and Practice, 8(4), 89-95. https://doi.org/10.5430/jnep.v8n4p89

Huizinga, J. (1972). Homo ludens. Alianza Editorial.

Kapp, K. (2012). The gamification of learning and instruction: game-based methods and strategies for training and education. Pfeiffer.

Kapp, K., Blair, L., y Mesch, R. (2014). The gamification of learning and instruction fieldbook: ideas into practice. Wiley.

Lee, J. J., y Hammer, J. (2011). Gamification in Education: What, How, Why Bother? Academic Exchange Quarterly, 15(2), 146-151. 
López-Pernas, S., Gordillo, A., Barra, E., y Quemada, J. (2019). Analyzing Learning Effectiveness and Students' Perceptions of an Educational Escape Room in a Programming Course in Higher Education. En IEEE Access, 7(1), 184221-184234. https://doi.org/10.1109/ACCESS.2019.2960312

Majuri, J., Koivisto, J., y Hamari, J. (2018). Gamification of Education and Learning: A Review of Empirical Literature. In Proceedings of the 2nd International GamiFIN conference (pp. 11-19). CEUR Workshop Proceedings.

Marín, I., y Hierro, E. (2013). Gamificación: el poder del juego en la gestión empresarial y la conexión con los clientes. Empresa Activa.

Martínez-Borobio, E., y Nalova-Katileva, E. (2020). L'escape room com a eina educativa per treballar la literatura a secundària: una proposta pràctica de Romeu i Julieta. REIRE Revista d'Innovació $i$ Recerca en Educació, 13(2), 1-18. https://doi.org/10.1344/reire2020.13.228965

McGonigal, J. (2011). Reality is broken: why games make us better and how they can change the world. Penguin Press.

Moreno Campos, V., y Suvires García, M. Á. (2018). Gamificación en la docencia de lingüística clínica: un Cluedo para los trastornos del lenguaje. @tic revista d'innovació educativa, 21, 49-56. https://doi.org/10.7203/attic.21.13365

Moura, A., y Lourido Santos, I. (2019). Escape room in education: Gamify learning to engage students and learn maths and languages. En B. D. Silva, J. A. Lencastre, M. Bento y A. J. Osório (eds.), Experiences and perceptions of pedagogical practices with game-based learning \& gamification (pp. 179-193). Centro de Investigação em Educação, Instituto de Educação da Universidade do Minho.

Murray, J. H. (2006). Toward a cultural theory of gaming: digital games and the co-evolution of media, mind, and culture. Popular Communication, 4(3), 185-202. https://doi.org/10.1207/s15405710pc0403_3

Nicholson, S. (2012). A user-centered theoretical framework for meaningful gamification. Games+Learning+Society 8.0. http://scottnicholson.com/pubs/meaningfulframework.pdf

Nicholson, S. (2015). Peeking behind the locked door: a survey of escape room facilities. http://scottnicholson.com/pubs/erfacwhite.pdf

Ortiz-Colón, A., Jordán, J. y Agredal, M. (2018). Gamificación en educación: una panorámica sobre el estado de la cuestión. Educação e Pesquisa, 44, e173773. https://doi.org/10.1590/s16784634201844173773

Peleg, R., Yayon, M., Katchevich, D., Moria-Shipony, M., y Blonder, R. (2019). Journal of chemical education, 96(5), 955-960. https://doi.org/10.1021/acs.jchemed.8b00406

Piaget, J. (1952). Play, dreams and imitation in childhood. Norton \& Co.

Piñero, J. C. (2019). Análisis sistemático del uso de salas de escape educativas: estado del arte y perspectivas de futuro. Espacios, 40(44), 1-20. 
Pujolà, J. T. (2019, 28 de mayo). Dos vías de escape: tendencias lúdicas en educación. UNIBA Centro Universitario Internacional de Barcelona.

https://www.unibarcelona.com/es/actualidad/noticias/dos-vias-de-escape-tendencias-ludicasen-educacion

Pujolà, J. T., Berríos, A., y Appel, C. (2017). Applying DMC in a gamified teacher course on gamification. En $\checkmark$ Congreso Internacional de Videojuegos y Educación CIVE 17. https://riull.ull.es/xmlui/handle/915/4769

Pujolà, J. T., y Herrera, F. (2018). Gamificación. En J. Muñoz-Basols, E. Gironzetti y M. Lacorte (eds.), The routledge handbook of spanish language teaching: metodologías, contextos y recursos para la enseñanza del español L2 (p. 583-595). Routledge.

Ramírez, J. L. (2014). Gamificación: mecánicas de juegos en tu vida personal y profesional. SC Libro.

Rodríguez, F., y Santiago, R. (2015). Gamificación: cómo motivar a tu alumnado y mejorar el clima en el aula. Digital text.

Sánchez, P. (2018). Escape rooms educativas: ejemplo práctico y guía para su diseño [Trabajo de Fin de Máster, Universitat Oberta de Catalunya]. http://openaccess.uoc.edu/webapps/o2/bitstream/10609/76505/6/patrisanlaTFM0118memoria. pdf

Santos, M. J., Miguel, M., Queiruga-Dios, A., y Hernández Encinas, A. (2019). Looking for the Antidote for Contaminated Water: Learning Through an Escape Room. En F. Martínez Álvarez, A. Troncoso Lora, J. Sáez Muñoz, H. Quintián y E. Corchado (eds.), International Joint Conference: 12th International Conference on Computational Intelligence in Security for Information Systems (CISIS 2019) and 10th International Conference on European Transnational Education (ICEUTE 2019). CISIS 2019, ICEUTE 2019. Springer.

Stone, Z. (2016, 28 de julio). The rise of educational escape rooms. The Atlantic. https://www.theatlantic.com/education/archive/2016/07/the-rise-of-educational-escaperooms/493316/

Veldkamp, A., Daemen, J. Teekens, S., Koelewijn, S., Knippels, M. C., y Van Joolingen, W. R. (2020). Escape boxes: Bringing escape room experience into the classroom. British Journal of Educational Technology, 51(4), 1220-1239. https://doi.org/10.1111/bjet.12935

Werbach, K., y Hunter, D. (2012). For the win: how game thinking can revolutionize your business. Wharton Digital Press.

Williams, P. (2018). Using escape room-like puzzles to teach undergraduate students effective and efficient group process skills. ISEC 2018 - Proceedings of the $8^{\text {th }}$ IEEE Integrated STEM Education Conference (pp. 254-257). https://doi.org/10.1109/ISECon.2018.8340495

Wu, C., Wagenschutz, H., y Hein, J. (2018). Promoting leadership and teamwork development through Escape Rooms. Medical Education, 52(5), 561-562. https://doi.org/10.1111/medu.13557 
La gamificación en juego: percepción de los estudiantes sobre un escape room educativo en el aula de español como lengua

extranjera

Yeasmin, S., y Abdulrahman, L. (2020). Escape The Countries: A VR Escape Room Game. $3^{\text {rd }}$ International Conference on Computer Applications \& Information Security (ICCAIS), Riyadh, Arabia Saudí. https://doi.org/10.1109/ICCAIS48893.2020.9096727 